\title{
EXACT SOLUTIONS FOR STEADY STATE, SPINE, AND FAN MAGNETIC RECONNECTION
}

\author{
I. J. D. CRAIG AND R. B. FABLing \\ University of Waikato, Hamilton, New Zealand \\ Received 1995 September 1; accepted 1995 November 6
}

\begin{abstract}
The problem of steady state, incompressible magnetic reconnection in three dimensions is addressed. It is shown that exact reconnection solutions can be constructed by superposing nonlinear disturbances onto three-dimensional magnetic $X$-points. There are two distinct families of reconnection solutions. These can be understood in terms of the eigenstructure of the null, that is, in terms of the "spine" curves and "fan" surfaces that define the separatrices of the field. One family of solutions is driven by disturbances in the fan and involves quasi-cylindrical current structures aligned to the axis of the spine; the other is associated with advection across the spine and a global current sheet aligned to the fan. Although both spine and fan solutions reduce to the two-dimensional analytic, shear-flow solutions of Craig \& Henton, the three-dimensional spine current formulation allows far richer reconnective current structures.

Subject headings: MHD - plasmas - Sun: activity
\end{abstract}

\section{INTRODUCTION}

It is well known that changes in magnetic field topology can only be effected by magnetic reconnection. Yet, despite intense theoretical investigation, the mechanisms underlying magnetic reconnection remain enigmatic. A recurring difficulty is achieving a significant reconnection speed given the low resistivities of astrophysical plasmas (e.g., Forbes \& Priest 1987; Biskamp 1994). More fundamentally, while two-dimensional planar reconnection appears to be well understood, at least physically, the three-dimensional picture remains cloudy. The problem is compounded by the absence of exact two- and three-dimensional global reconnection solutions.

Historically, $X$-type neutral points have provided the raw material of magnetic reconnection theory (e.g., Petschek 1964). The conventional picture of planar reconnection involves the advection of material across identical separatrix planes, the field lines being cut and rejoined at the center of the $X$-point. Analytic models of this process are provided by linear $X$-point theory (Craig \& McClymont 1991, 1993; Hassam 1992). It is suspected, however, that more complicated flow topologies are required in the presence of three-dimensional neutral points (e.g., Rosenau 1979). Certainly, the eigenstructure of magnetic nulls changes significantly in the transition from even to odd dimensionalities (e.g., Lau \& Finn 1990). One separatrix plane of the two-dimensional $X$-point is lost in the transition to three-dimensions. What remains is a single separatrix plane plus a unique field line which threads the neutral point. Following Priest \& Titov (1995), we call these the "fan" and "spine curve," respectively. The question is: how can spine and fan structures be extrapolated from twodimensional models that maintain strict parity between each separatrix surface?

The purpose of this paper is to demonstrate that exact families of three-dimensional magnetic reconnection solutions can be constructed analytically by extending the planar analysis of Craig \& Henton (1995). We note that Craig \& Henton derive an exact, nonlinear solution for incompressible reconnection by breaking the conventional planar symmetries. We show that an extension into three dimensions involves two classes of solutions: in one class, nonlinear distortions of the fan lead to reconnection by quasi-cylindrical current structures along the spine; in the other, advection across the spine leads to global current sheets aligned to the fan.

The paper is organized as follows. In $\S 2$ we review briefly the geometry of $X$-type neutral points in two and three dimensions. Section 3 describes the reconnection equations and outlines our method of solution. Our central results concerning spine and fan current reconnection are derived in $\S 4$. We demonstrate, in particular, that analytic planar solutions (Craig \& Henton 1995) can be derived by invoking either the fan or spine formulations. Our conclusions are presented in $\S 5$.

\section{THE THREE-DIMENSIONAL RECONNECTION TOPOLOGY}

\subsection{Geometry of Null Points}

We begin by considering the field line topology close to a null point of the field $\boldsymbol{B}(\boldsymbol{x})$. We expand the magnetic field in the neighborhood of the point $\boldsymbol{x}=\boldsymbol{x}_{0}$ (say) according to

$$
B_{i}\left(x_{0}+d x\right)=B_{i}\left(x_{0}\right)+B_{i, j}\left(x_{0}\right) d x_{j},
$$

where $B_{i, j}$ is an element of the Jacobian matrix $\partial \boldsymbol{B} / \partial \boldsymbol{x}$. The constraint $\boldsymbol{\nabla} \cdot \boldsymbol{B}=0$ then implies the trace condition $B_{i, i}=0$. Obviously, the current density at $x_{0}$, namely,

$$
J_{i}=\epsilon_{i j k} B_{k, j},
$$

vanishes identically only in the case of symmetric $B_{k, j}$.

Suppose now that $\boldsymbol{x}_{0}$ locates a null point of the field. Nulls can be classified according to the geometry of the field lines threading the neutral point; in other words, in terms of separatrices isolating topologically distinct regions of magnetic flux. The differential field line equations $\gamma d x=B$ give

$$
\left(B_{i, j}-\gamma I_{i j}\right) d x_{j}=0,
$$

where $I$ is the identity matrix, and so the eigenvectors $d x_{j}$ determine field line directions in the vicinity of the null. The 
eigensystem is generally complex, but the eigenvalues satisfy

$$
\gamma_{1}+\gamma_{2}+\gamma_{3}=0 \text {, }
$$

by virtue of the trace condition $B_{i, i}=0$. Since $B_{i, j}$ is real, complex eigenvalues can only occur in conjugate pairs.

\section{2. $X$-Type Null Points}

Detailed discussions of the nature of magnetic nulls have already been given in the literature (e.g., Lau \& Finn 1990) and we present only a brief discussion here. In twodimensional geometries, the nulls are either $X$-type or $O$-type depending on whether the eigenvalues are real or complex. The prototype for planar reconnection is the current-free $X$-point whose eigensystem is real and corresponds to separatrix planes inclined at $\pm 45^{\circ}$ to the $x$-axis. In contrast, the $O$-point has a complex eigenstructure: there are no separatrix lines, only circular field lines surrounding the null.

In three dimensions, there are natural extensions of planar $X$ - and $O$-point structures. Of central interest is the $X$-point topology. The eigenstructure is real, and eigenvalues of like sign possess eigenvectors which form a plane through the neutral point. This plane, the separatrix fan, contains all field lines emanating radially from the null. The remaining eigenvector defines a single line, the spine curve, which threads the null perpendicular to the surface of the fan.

What are the physical implications of these results? Certainly, in the absence of resistivity, it is known that perturbations of the $X$-point can lead to current singularities along the separatrices (Lau \& Finn 1990; Priest \& Titov 1995). Such behavior is often taken as a signature of threedimensional reconnection, though, strictly speaking, magnetic annihilation may be sufficient to resolve the singularity (e.g., Biskamp 1994). In what follows, we show that the underlying forms of three-dimensional magnetic reconnection are implied by the eigensystem of the $X$-point.

\subsection{Two- and Three-dimensional $X$-Point Reconnection}

In planar reconnection modeling, it is natural to impose symmetries which reflect the underlying $X$-point topology. Under these "traditional" symmetries, only one quadrant of the reconnection region need be modeled (e.g., Forbes \& Priest 1987).

But we have already seen that the three-dimensional $X$-point topology involves only a single separatrix plane. It follows that, in the transition from two to three dimensions, one separatrix surface must collapse into a spine curve, in much the same way as a planar null line degenerates into a three-dimensional null point. It is hard to see how this can occur under two-dimensional reconnection symmetries which impose parity between each separatrix surface.

Notably, in the only exact two-dimensional reconnection solution to date, there is a breakdown of the traditional reconnection symmetries (Craig \& Henton 1995). The model involves the flow of material across one separatrix plane only: the other is contiguous with a global current sheet but has no flow across it. Thịs solution generalizes naturally into three dimensions.

\section{STEADY STATE RECONNECTION EQUATIONS}

\subsection{Momentum and Induction Equations}

We consider the steady state momentum and induction equations for an incompressible inviscid plasma. These are written conveniently in the nondimensional form

$$
\begin{gathered}
(v \cdot \nabla) \omega-(\omega \cdot \nabla) v=(B \cdot \nabla) J-(J \cdot \nabla) B, \\
(v \cdot \nabla) B-(B \cdot \nabla) v=\eta \nabla^{2} B,
\end{gathered}
$$

where the magnetic and velocity fields satisfy

$$
\boldsymbol{\nabla} \cdot \boldsymbol{B}=0, \boldsymbol{\nabla} \cdot \boldsymbol{v}=0,
$$

and the current density and fluid vorticity are given by

$$
\boldsymbol{J}=\boldsymbol{\nabla} \times \boldsymbol{B}, \boldsymbol{\omega}=\boldsymbol{\nabla} \times \boldsymbol{v} .
$$

The remarkable feature of this system is the symmetry in the $\boldsymbol{v}$ and $\boldsymbol{B}$ fields. This is broken only by the resistive term which provides an energy sink for the system. The fact that the dimensionless resistivity $\eta$ is typically very small, of order $10^{-10}$, implies that resistive effects can be significant only in localized regions of high current density. However, since resistive dissipation is a key ingredient of magnetic reconnection, it appears that a collapse to small length scales must occur if rapid changes in the global field topology are to be achieved.

\subsection{Analytic Forms for Spine and Fan Reconnection}

The reconnection problem is nonlinear, but solutions can be constructed by exploiting the symmetry in the $\boldsymbol{B}$ and $\boldsymbol{v}$ fields. Following Craig \& Henton (1995), we consider solutions constructed by superposing some nonlinear "displacement field " onto background potential field solutions.

The simplest potential field of interest has the stagnation point form

$$
\boldsymbol{P}(\boldsymbol{x})=\alpha[-x \hat{\boldsymbol{x}}+\kappa y \hat{\boldsymbol{y}}+(1-\kappa) z \hat{z}],
$$

where $\alpha$ and $\kappa$ govern the strength and isotropy of the field. We can assign a special role to the $x$-axis by taking $0 \leq \kappa \leq 1$. If $\boldsymbol{P}$ is identified as a velocity field, then all planes $|x|>0$ correspond to material inflow for positive $\alpha$. Interpreted as a magnetic field, $\boldsymbol{P}$ can be identified as a threedimensional $X$-point. The $x$-axis defines a spine curve into the null $(\alpha>0)$, and the fan comprises all outgoing field lines in the plane $x=0$. The eigenvalues $\gamma_{i}$ form the set $\alpha[-1, \kappa,(1-\kappa)]$, and the special case $\kappa=\frac{1}{2}$ corresponds to rotational symmetry about the $x$-axis. Either $y$ or $z$ can be made ignorable by the choice $\kappa=0$ or $\kappa=1$, respectively.

Of course, potential field solutions are of little interest in problems of magnetic reconnection. To construct more general solutions, we consider field superpositions of the generic form $\boldsymbol{P}(\boldsymbol{x})+\boldsymbol{Q}(\boldsymbol{x})$, where

$$
\boldsymbol{Q}(\boldsymbol{x})=X(y, z) \hat{\boldsymbol{x}}+Y(x, z) \hat{\boldsymbol{y}}+Z(x, y) \hat{z} .
$$

Although $Q(x)$ has no directional asymmetry, an inherent "grain" has been imposed on the problem by the representation of $\boldsymbol{P}$. Specifically, since $x$ is a nonignorable coordinate, there is a unique one-variable form for $\boldsymbol{Q}$, namely,

$$
\boldsymbol{Q}(x)=Y(x) \hat{\boldsymbol{y}}+Z(x) \hat{z} .
$$

We know from Craig \& Henton (1995) that of all the potential fields, equation (3.5) is the only background field that admits superpositions of the form $\boldsymbol{P}+\boldsymbol{Q}(x)$. The analysis of Craig et al. (1995) shows that equation (3.7) leads to reconnection by currents in the fan surface $x=0$.

There is, however, a two-variable analog to equation (3.7), namely,

$$
\boldsymbol{Q}(y, z)=X(y, z) \hat{\boldsymbol{x}} .
$$


As shown below, this form leads to reconnection by currents along the spine.

Finally, we remark that more general disturbancesformed, say, by the superposition of equations (3.7) and (3.8)--are forbidden by the nonlinearity of the reconnection system.

\section{STEADY STATE RECONNECTION SOLUTIONS}

\subsection{Introduction}

First we discuss spine reconnection using the displacement field of equation (3.8). Both Cartesian and cylindrical forms of the spine reconnection equations are employed (in $\$ 4.2$ and 4.3 , respectively). Fan reconnection solutions are discussed in $\S 4.4$. In $\S 4.5$, we illustrate how twodimensional reconnection models can be derived using either the fan or spine formulations. The features common to all these reconnection solutions are summarized in $\S$ 4.6.

\subsection{Solution for Spine Current Reconnection}

Suppose equation (3.8) is interpreted as a velocity fieldsay $v=v(y, z) \hat{x}$. Then flow is imposed uniformly on all planes of constant $x$. There are disturbances in the fan, but the spine remains undistorted.

The solution is obtained by substituting $\boldsymbol{v}=\boldsymbol{P}+\boldsymbol{q}(y, z)$ and $\boldsymbol{B}=\lambda \boldsymbol{P}+\boldsymbol{Q}(y, z)$ into the momentum and induction equations. We obtain

$$
\boldsymbol{v}=\boldsymbol{P}+\lambda X(y, z) \hat{\boldsymbol{x}}, \quad \boldsymbol{B}=\lambda \boldsymbol{P}+X(y, z) \hat{\boldsymbol{x}},
$$

where $X$ satisfies

$$
\alpha\left(1-\lambda^{2}\right)\left[X+\kappa y X_{y}+(1-\kappa) z X_{z}\right]=\eta \nabla^{2} X .
$$

Equation (4.2) is the basic equation for spine reconnection. In what follows, we shall simplify the discussion by assuming that the sign of $\alpha\left(1-\lambda^{2}\right)$ is determined by $\alpha$. This is equivalent to restricting the curvature of the field lines according to $|\lambda|<1$.

It is readily seen that singularities develop in the absence of resistive effects. Suppose, for instance, that $X=X_{0}(y)$ on the line $z=1$ (say). Then, by the method of characteristics, we obtain a solution

$$
X(y, z)=z^{1 /(\kappa-1)} X_{0}\left(y z^{\kappa /(\kappa-1)}\right), \quad \eta=0,
$$

which is clearly unbounded on $z=0$.

Obviously resistive diffusion is required to resolve the singularity. It is easy to verify that well-behaved particular solutions can be derived by assuming either multiplicative or additive separability, that is, $X=f(y) g(z)$ or $X=f(y)+g(z)$. Although general solutions can be constructed by superposition, we find it more instructive to consider cylindrical mode solutions for the case of axisymmetric background fields.

\subsection{Cylindrical Spine Current Reconnection}

We assume $\kappa=\frac{1}{2}$ and employ cylindrical coordinates $(r$, $\theta)$ in the planes $x=$ const. Then $\boldsymbol{P}$ takes the form

$$
\boldsymbol{P}(x, r)=\alpha\left(-x \hat{\boldsymbol{x}}+\frac{1}{2} r \hat{\boldsymbol{r}}\right),
$$

and equation (4.2) reduces to

$$
\alpha\left(1-\lambda^{2}\right)\left(X+\frac{1}{2} r X_{r}\right)=\eta \nabla^{2} X .
$$

The solution for vanishing $\eta$ is seen to be

$$
X(r, \theta)=\frac{A(\theta)}{r^{2}}, \quad \eta=0,
$$

which confirms that resistive diffusion is required to resolve the singularity along the spine.

We now exploit the azimuthal symmetry by taking

$$
X(r, \theta)=f(r) e^{i m \theta} .
$$

Equation (4.5) reduces to

$$
\alpha\left(1-\lambda^{2}\right)\left(f+\frac{1}{2} r f^{\prime}\right)=\eta\left(f^{\prime \prime}+\frac{f^{\prime}}{r}-\frac{m^{2} f}{r^{2}}\right),
$$

and it follows that the solution for small $r$ has the form

$$
f(r)=f_{m} r^{m}+O\left(r^{m+2}\right) .
$$

Since the current density is given by

$$
J(r, \theta)=\left[\operatorname{im} \frac{f(r)}{r} \hat{\boldsymbol{r}}-f^{\prime}(r) \hat{\boldsymbol{\theta}}\right] e^{i m \theta},
$$

there can only be finite current at the origin if $m=1$. It follows that only the $m=1$ mode corresponds to topological reconnection. All other modes are associated with pure ohmic dissipation.

Of course, the current density must also be bounded at large $r$. Dimensional considerations imply that the diffusion region is limited to a thin cylinder of radius $r \sim \eta^{1 / 2}$ surrounding the spine. Outside this region, we expect that the field should fall off as $r^{-2}$, in accordance with equation (4.6). In fact, all modes except $m=0$ conform to this behavior.

A discussion of the spine reconnection equation is given in the Appendix, where it is shown that closed form solutions can be derived for all even modes. In addition, we point out that all modes $m>0$ can be approximated using boundary layer expansions for the diffusion region. The exceptional $m=0$ solution is given by

$$
f(r)=f_{0} \exp \left(-\frac{\mu^{2} r^{2}}{2}\right), \quad \mu^{2}=-\frac{\alpha\left(1-\lambda^{2}\right)}{2 \eta}, \quad m=0 .
$$

The field is finite at the origin but falls off exponentially with distance provided that $\alpha<0$, corresponding to inflow in the fan but outflow along the spine. This mode is unique in that the neutral point is displaced along the spine, resulting in flow through the null of the field. It is also the only mode that allows a net displacement (averaged over $\theta$ ) of the fan. Although the inner field decays over the $\eta^{1 / 2}$ length scale, the violation of equation (4.6) suggests that diffusion is important globally, and not just restricted to a small boundary layer surrounding the spine. Figure 1 illustrates the magnetic field structure for the $m=0$ solution.

Consider now the reconnective $m=1$ solution. The field vanishes at the origin, in common with all other modes $m>1$, but the current density is maximum along the spine. Figure $2 a$ shows how the radial structure of the field can be approximated by matching the outer field solution (4.6) to an inner expansion of the diffusion region (as described in the Appendix). The current density, shown in Figure $2 b$, is localized over the length scale $r \sim \eta^{1 / 2}$ but falls off rapidly, as $r^{-3}$, with increasing radius. Since the amplitude of the field builds up as $\eta^{-1}$ (assuming fixed conditions on $r=1$, 


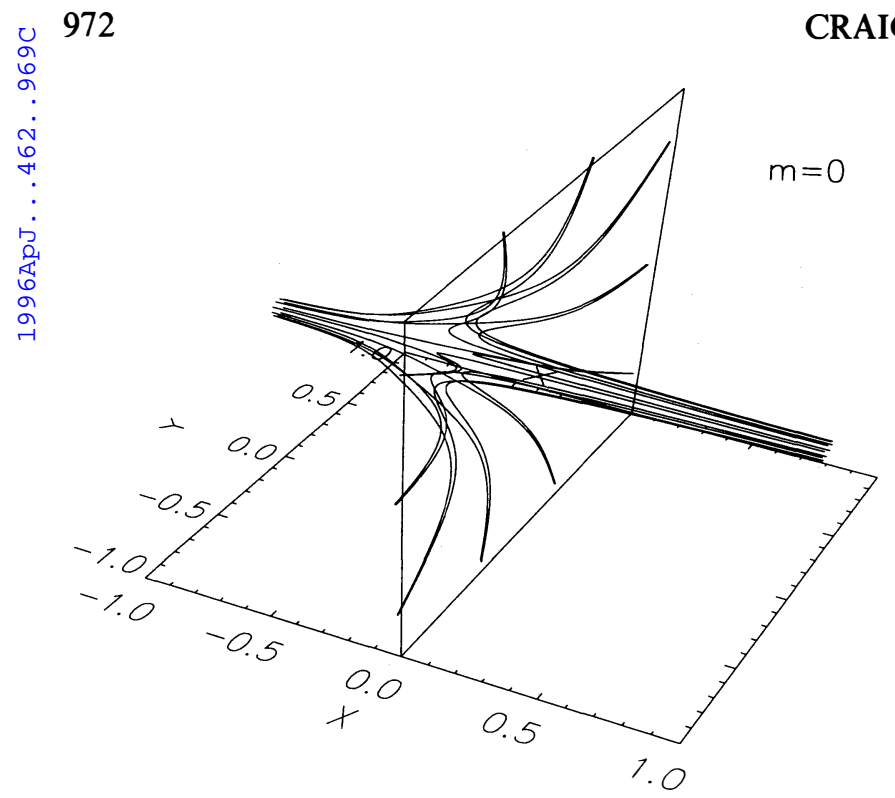

Fig. 1.-Distortion of the equilibrium fan surface $(x=0)$ by the perturbation field $X$. For $m=0$, the displacement field peaks on the spine axis, and so the null of the field is displaced from the origin. Parameters are $\alpha=-2, \lambda=0.9$, and $\eta=0.01$.

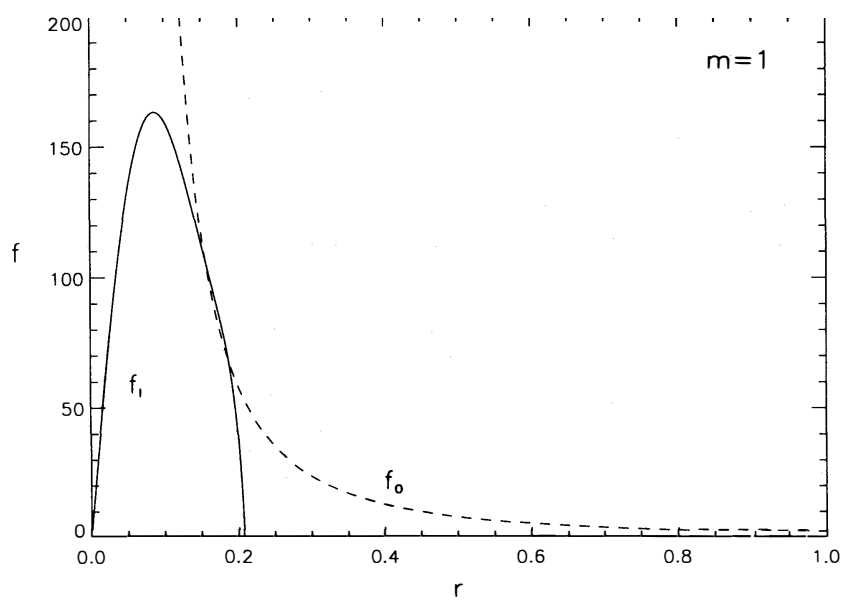

FIG. $2 a$ say), the current density increases as $\eta^{-3 / 2}$. This guarantees fast reconnection (see $\S 4.6$ ).

Close to the spine axis, we note that

$$
J(r, \theta)=J_{0}(\sin \theta \hat{r}-\cos \theta \hat{\boldsymbol{\theta}}), \quad m=1,
$$

that is, $\boldsymbol{J}=J_{0} \hat{z}$ (to within an arbitrary phase). Figure $2 c$ shows how the current density resembles two counterrotating tubes aligned to the spine. Although similar current structures are present for $m>1$ (there are $m$ pairs of counterrotating tubes), the current density on the spine always vanishes for $m \neq 1$. The field line structure of the reconnective mode is shown in Figure 3.

Finally, in Figure 4, we show surfaces of the displacement field $X(r, \theta)$ for the cases $m=0,1,2,3$. Apart from $m=0$, all displacement fields build up rapidly across the inner diffusive layer $r \leq \eta^{1 / 2}$. Outside this layer, all fields ( $\left.m \geq 1\right)$ decay at the asymptotic rate $r^{-2}$. Since the field line equations are given by

$$
\frac{d x}{-\alpha \lambda x+X(r, \theta)}=\frac{d r}{(1 / 2) \alpha \lambda r},
$$

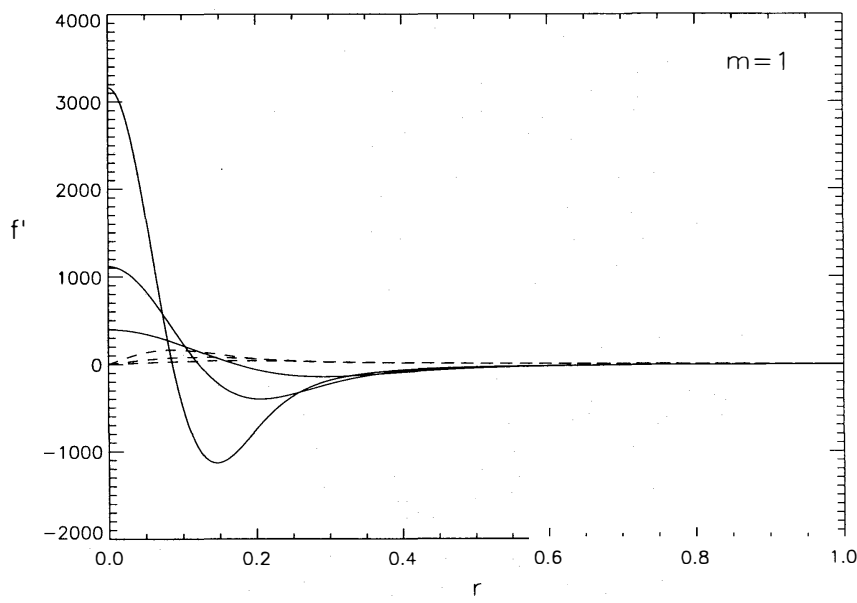

FIG. $2 b$

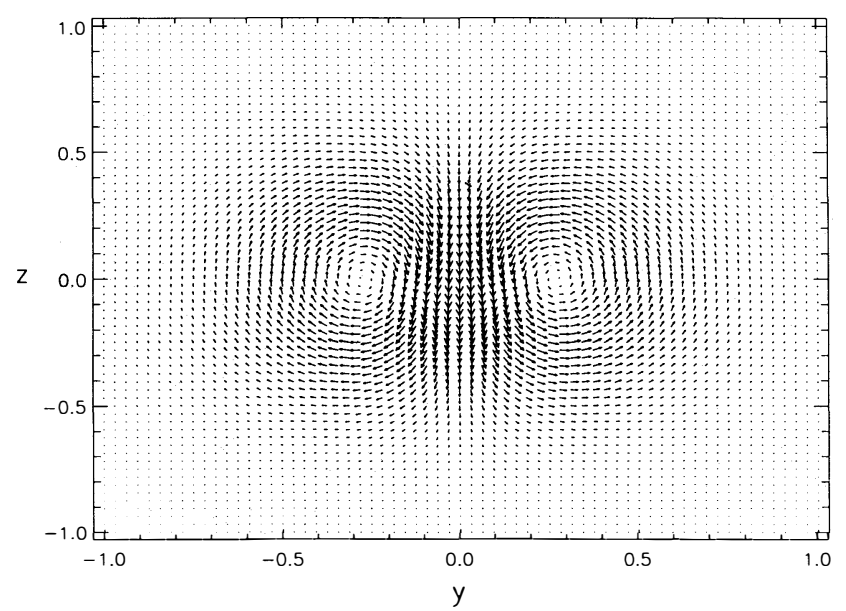

Fig. $2 c$

FIG. 2.-(a) Plot of the displacement field amplitude $f(r)$ for $m=1$ spine reconnection. The inner solution $f_{I}$ determined by an eight-term series expansion intersects the outer solution $f_{0}=A / r^{2}$. The exact solution is visually indistinguishable from the approximation obtained by matching $f_{I}(r)$ to $f_{0}(r)$. Solution parameters are $\alpha=-2, \lambda=0.9$, and $\eta=0.001$. (b) The buildup in current density as $\eta$ is reduced for the $m=1$ mode. The factor of 8 increase is a reflection of the steepening field gradient $f^{\prime}(r)$ (solid line). By contrast, the field buildup is relatively weak (dashed line). Solution parameters are $\alpha=-2, \lambda=0.9$, and $\eta=0.004,0.002,0.001$. (c) Vector plot of the current density for $m=1$ spine reconnection. The current density falls off rapidly with increasing distance from the spine axis and resembles two counterrotating tubes aligned to the spine. Parameters are the same as in Fig. 1. 


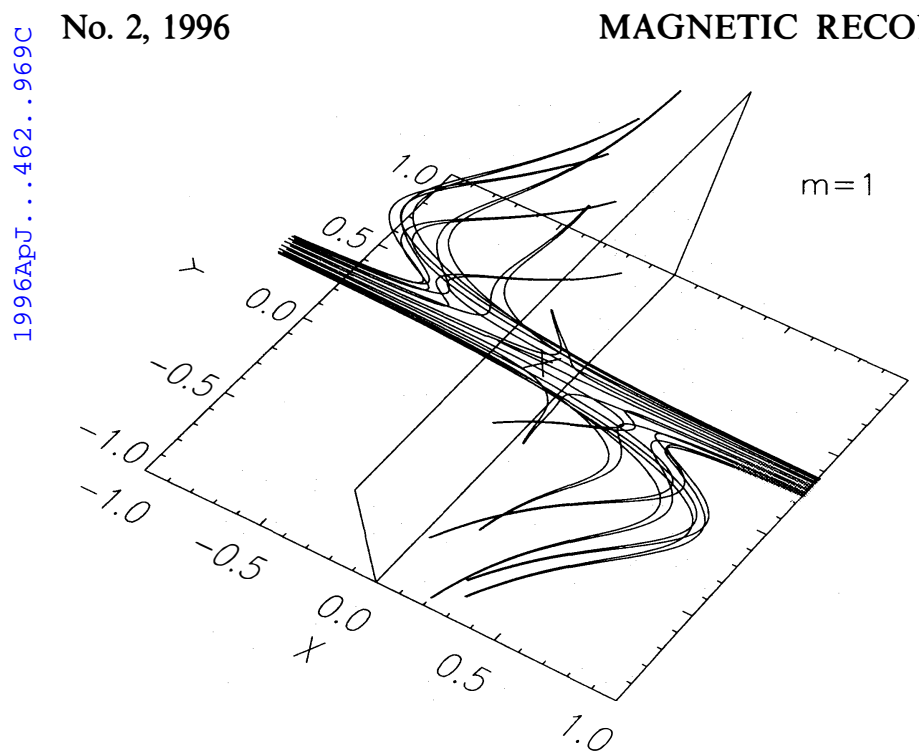

FIG. 3.-Shearing of the fan surface in the reconnective $m=1$ mode Field lines are reconnected as they advect across the fan surface. Parameters are the same as in Fig. 1.

we note that $d x=0$ corresponds to a turning point of a given field line. A particular value of the turning point, $x=x_{p}$ (say), defines a locus of points for which $X(r, \theta)=$ $\alpha \lambda x_{p}$. Thus, $X(r, \theta)$ provides a measure of the field line penetration through the fan.

\subsection{Fan Current Reconnection}

Now we consider the displacement field of equation (3.7). Interpreted as a magnetic field, this form corresponds to straight field lines whose intensity and orientation vary with $x$. Accordingly, it is the spine curve of $\boldsymbol{P}$, rather than the fan, which is distorted by the displacement field $Q(x)$. The result is reconnection driven by currents in the fan.
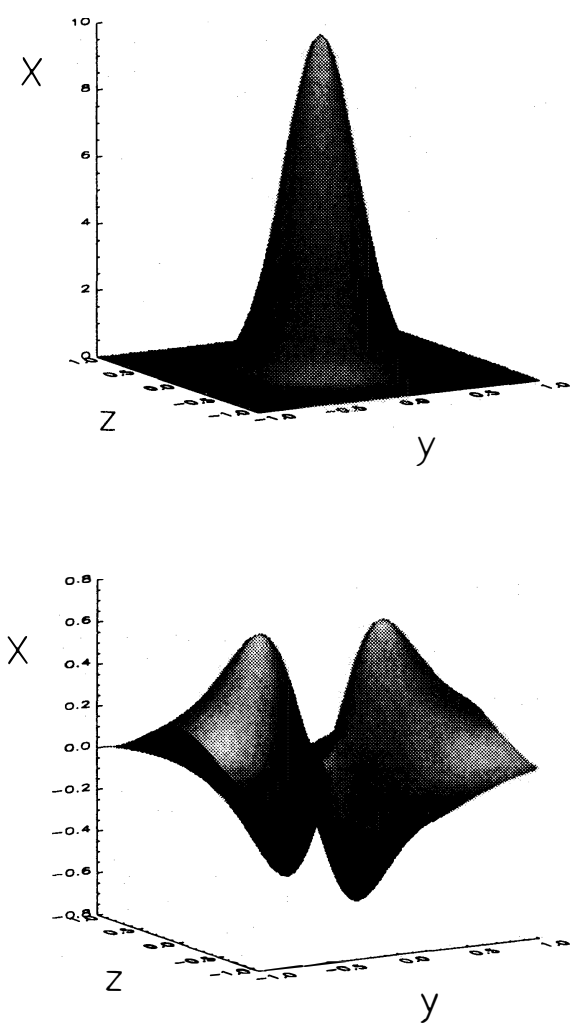

The solution is given by

$$
\boldsymbol{v}=\boldsymbol{P}+\lambda \boldsymbol{Q}(x), \quad \boldsymbol{B}=\lambda \boldsymbol{P}+\boldsymbol{Q}(x),
$$

where $\boldsymbol{Q}=Y(x) \hat{\boldsymbol{y}}+Z(x) \hat{z}$ is subject to

$$
\begin{gathered}
\alpha\left(1-\lambda^{2}\right)\left(\kappa Y+x Y^{\prime}\right)+n Y^{\prime \prime}=0, \\
\alpha\left(1-\lambda^{2}\right)\left[(1-\kappa) Z+x Z^{\prime}\right]+n Z^{\prime \prime}=0 .
\end{gathered}
$$

A discussion of this solution has already been given by Craig et al. (1995), and we shall summarize only the salient features. The outer field solutions, $Y \sim x^{-\kappa}, Z \sim x^{\kappa-1}$, are valid outside the diffusion layer $|x|>O\left(\eta^{1 / 2}\right)$. The inner solution is dominated by a global current sheet aligned to the fan surface $x=0$ across which there is no flow. Reconnection occurs when fluid particles are advected across the spine curve. Close to the neutral point, the spine has the linear form $y=-a_{1} x /(1+\kappa), z=-a_{2} x /(2-\kappa)$, but for large $x$ the spine is contiguous with the $x$-axis. The case $\lambda=0$ is degenerate (it corresponds to the stagnation flow, annihilation model of Sonnerup \& Priest 1975), but, more generally, shear flows develop allowing curved field lines to reconnect across the neutral point.

Figure 5 illustrates the separatrix field lines for various values of $\kappa$. The solution for $\kappa=\frac{1}{2}$ has radial field lines in the fan, but curvature develops as $\kappa$ is reduced. In the limit $\kappa \rightarrow 0$, the solution is near-planar, but separatrix field lines are still "channeled" through the null.

\subsection{Planar Reconnection Solutions}

We can obtain planar reconnection solutions from both spine and fan current formulations. Consider first the spine formulation of equation (4.2). On taking $\kappa=1$ and assuming that $z$ is ignorable, we obtain

$$
E+\alpha\left(1-\lambda^{2}\right) X=\eta X^{\prime}, \quad X=X(y),
$$
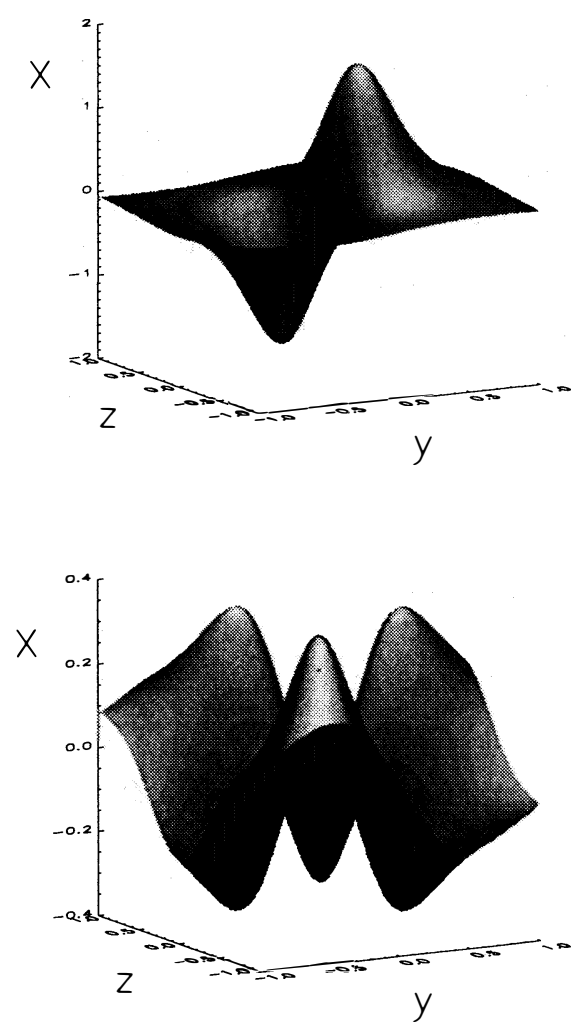

Fig. 4. - Surfaces of the displacement field for the $m=0,1,2,3$ modes. Parameters are the same as in Fig. 1 . 


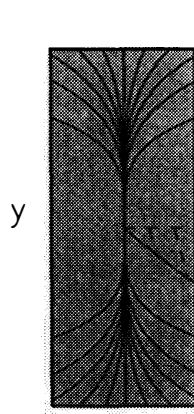

Z

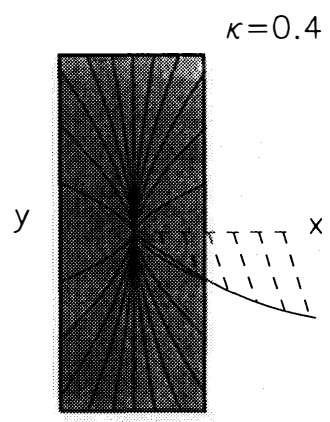

Z

$\kappa=0.2$

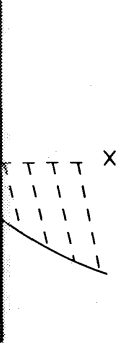

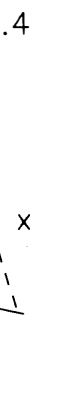

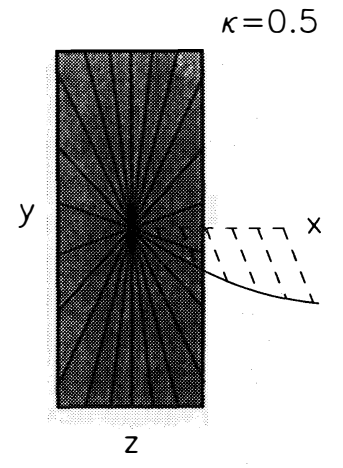

Fig. 5.-Separatrix field lines for fan-current reconnection in the cases $\kappa=0.2,0.3,0.4,0.5$. Solution parameters are $\eta=0.05, \alpha=2, \lambda=0.9$, and $E=0.1$.

where $E$ is a constant of integration. The solution can be expressed in terms of the Dawson integral, via

$$
Q(y)=\frac{E}{\eta \mu} \operatorname{daw}(\mu y) \hat{x}, \quad \operatorname{daw}(s)=\int_{0}^{s} \exp \left(t^{2}-s^{2}\right) d t,
$$

where $\mu^{2}=-(\alpha / 2 \eta)\left(1-\lambda^{2}\right)$. This is just the planar reconnection solution of Craig \& Henton (1995) with outflow along the $x$-axis (since we must take $\alpha<0$ ). Reconnection takes place when fluid is advected across a separatrix "flow" plane which intersects the current plane at the angle $2 \alpha \lambda \eta / E$. The width of the current layer is governed by the behavior of daw $(\mu y)$. The disturbance field $Q(y)$ piles up at the edge of the diffusion layer $y \sim O\left(\eta^{1 / 2}\right)$ but diminishes rapidly, as $1 /(2 \mu y)$, for larger $y$. The fact that $E$ can be chosen independently of $\eta$ without upsetting the external field conditions at large $y$ qualifies the reconnection solution as "fast" (Craig \& Henton 1995). However, it seems likely that the reconnection rate must ultimately be limited by external factors, for instance, the magnitude of magnetoconvective forces driving the merging (see $\S 4.6$ ).

Slightly more general planar models can be constructed using the fan current formulation of $\S 4.4$. Choosing $z$ as the ignorable coordinate (i.e., $\kappa=1$ ) yields the explicit form

$$
\boldsymbol{Q}(x)=\frac{E}{\eta \mu} \text { daw }(\mu x) \hat{\boldsymbol{y}}+\left[\frac{\sqrt{\pi}}{2 \mu} Z^{\prime}(0) \operatorname{erf}(\mu x)+Z(0)\right] \hat{z},
$$

where $\mu^{2}=(\alpha / 2 \eta)\left(1-\lambda^{2}\right)$ and we take $\alpha>0$. Apart from the reversed velocity field, this solution is physically identical to that of equation (4.18) in the case $Z(0)=Z^{\prime}(0)=0$. Obviously, by allowing $Z(0)$ and $Z^{\prime}(0)$ to be nonzero both uniform and highly sheared $z$-components can be superposed onto the intrinsic planar solution. In contrast to the Dawson function behavior, the error function solution is not associated with flux pileup at the onset of the current layer. In the case $|Z(0)|>0$, planar reconnection occurs in the absence of a null point in the field.

Finally, we note that the planar solutions described above can be generalized slightly by superposing an external shear flow onto the solution (Craig \& Henton 1995). Such external flows are forbidden in the fully threedimensional solutions (Craig et al. 1995).

\subsection{Discussion}

It is clear that the previous reconnection solutions have the generic form

$$
\boldsymbol{v}=\boldsymbol{P}+\lambda \boldsymbol{Q}, \quad \boldsymbol{B}=\lambda \boldsymbol{P}+\boldsymbol{Q},
$$

with $\lambda$ nonzero. For spine reconnection, the vector field $\boldsymbol{Q}$ has a two-variable form $Q=X(y, z) \hat{\boldsymbol{x}}$ aligned to the direction of the spine. Fan reconnection, however, is defined by the one-variable form $\boldsymbol{Q}=Y(x) \hat{\boldsymbol{y}}+Z(x) \hat{z}$, whose direction is perpendicular to the $x$-axis.

The plasma pressure associated with spine reconnection is given by

$$
p(x, y, z)=p_{0}-\frac{1}{2}\left(P^{2}+X^{2}\right)+\alpha \lambda x X,
$$

where $\boldsymbol{P}$ is given by equation (3.5). For fan reconnection, we have that

$$
p(x, y, z)=p_{0}-\frac{1}{2}\left(P^{2}+Y^{2}+Z^{2}\right)-\alpha \lambda[\kappa y Y+(1-\kappa) z Z] .
$$

The fact that all solutions maintain fast dissipation by piling up flux at the onset of the diffusion region places stringent requirements on the plasma pressure. If we exclude the exceptional behavior of the $m=0$ spine mode, then all fields build up according to $Q_{M} \sim \eta^{-\beta}$, where $\frac{1}{4} \leq \beta \leq 1$. Clearly, to maintain positive pressures, $p_{0}$ must increase as $Q_{M}^{2} \sim \eta^{-2 \beta}$.

For example, the spine solutions scale as $Q_{M} \sim \eta^{-1}$ for $m \geq 1$. The weakest buildup is associated with $\kappa=\frac{1}{2}$ fan current reconnection. The pressure buildup is relatively modest, $p_{0} \sim \eta^{-1 / 2}$, but a fast ohmic dissipation rate $W_{\eta} \sim$ $\eta J^{2} \Delta x \sim \eta^{0}$ is still maintained by virtue of the scalings $J \sim \eta^{-3 / 4}, \Delta x \sim \eta^{1 / 2}$. More generally, the ohmic dissipation rate is superfast; that is, $W_{\eta}$ increases with reductions in the plasma resistivity!

On physical grounds, however, the flux pileup must reflect the magnitude of external forces driving the reconnection. For instance, if footpoint motions drive the merging, then $Q_{M}$ may be identified with the magnitude of fields at the base of the solar atmosphere. Either way, since the magnetoconvention forces powering the merging cannot increase indefinitely, there must be a physical limit to the flux pileup that can be achieved. To what extent this limits the reconnection speed is unclear, but obviously arbitrarily fast merging in the limit of small $\eta$ is not permitted.

\section{CONCLUSIONS}

We have constructed exact solutions for steady state, incompressible magnetic reconnection in three dimensions. All solutions can be naturally interpreted in terms of the separatrix structure of the field, as defined by the spine and fan eigenstructure close to the null point. In particular, we 
have shown that motions across the plane of the fan lead to quasi-cylindrical current structures aligned to the spine, in other words, to spine current reconnection. By the same token, advection across the spine is associated with a global current sheet aligned to the fan.

Both spine and fan formulations naturally reproduce the analytic, two-dimensional, shear flow solutions of Craig \& Henton (1995). A key feature of the two-dimensional solution is a breakdown of the strict $X$-point symmetry traditionally associated with planar reconnection models. Only one separatrix plane has flow across it; the other is aligned to a global current sheet (of width $\eta^{1 / 2}$ ) across which there is no flow. Fast reconnection is maintained by the pileup of flux at the onset of the sheet.

Conversely, the morphology of the three-dimensional solution can be anticipated from the planar analysis. Whether spine or fan reconnection is achieved depends upon which of the two-dimensional separatrix planes collapses into the spine. This is governed, in the present analysis, by the form of the nonlinear disturbance field $\boldsymbol{Q}$. For instance, in spine reconnection, the current plane collapses but the advection plane is preserved as the fan. What remains are tubes of current aligned to the spine axis, and it is the distribution of current over the spine that determines whether reconnection occurs. Thus, in the axisymmetric case, it is only the $m=1$ azimuthal mode that allows reconnection, all other modes having vanishing currents at the neutral point. By contrast, a much simpler current structure is associated with fan reconnection, namely, a onedimensional current sheet overlying the neutral point.

In summary, for isolated $X$-points we have argued that spine and fan reconnection provide the archetypal forms for steady state magnetic merging in three dimensions. Of course, in more general circumstances-for example, in dynamic compressible plasmas-we would not expect "pure" spine or fan reconnection to occur. In such cases, it seems likely that hybrid forms of reconnection may evolve in which localized currents are distributed over all the separatrices of the field.

It is a pleasure to acknowledge helpful discussions with Paul Watson, Alfred Sneyd, and Stefan Henton. The financial support of a FORST research grant is gratefully acknowledged.

\section{APPENDIX}

\section{THE CYLINDRICAL SPINE EQUATION}

\section{A1. BOUNDARY LAYER ANALYSIS}

Suppose we consider equation (4.8) on the assumption that the resistive term

$$
\eta\left(f^{\prime \prime}+\frac{f^{\prime}}{r}-\frac{m^{2}}{r^{2}} \cdot f\right)=0
$$

determines an inner solution, valid for small $r$. Solutions which remain finite at the origin are given by

$$
f_{I}(r)=r^{m} \text {. }
$$

The outer solution $f_{0}(r)=A / r^{2}$ (eq. [4.6]) is obtained by solving

$$
f+\frac{1}{2} r f^{\prime}=0 \text {. }
$$

This is just the spine equation in the case $\eta=0$.

The inner solution (A2) implies that equation (A3) has a contribution of order $r^{m}$ as $r \rightarrow 0$. The fact that equation (A3) maintains a finite contribution for $m=0$ implies that the inner and outer approximations break down for this mode.

\section{A2. SERIES SOLUTIONS}

If we write the spine equation (4.8) in the form

$$
x^{2} f^{\prime \prime}+\left(x+\frac{1}{2} x^{3}\right) f^{\prime}+\left(x^{2}-m^{2}\right) f=0,
$$

where

$$
f=f(x), \quad x=\left[\frac{\eta}{\alpha\left(\lambda^{2}-1\right)}\right]^{-1 / 2} r
$$

and assume the series expansion

$$
f(x)=x^{v} \sum_{l=0}^{\infty} a_{l} x^{l}
$$

we obtain the two-term recurrence relation

$$
a_{l+2}=a_{l} \frac{1+(1 / 2)(v+l)}{m^{2}-(v+l+2)^{2}}, \quad v= \pm m,
$$

where $a_{0}=1, a_{1}=0$. In the case $v=-m$, the series terminates for even $m$. Given these particular integrals, $p(x)$ say, we can 
deduce general solutions, for example,

$$
p(x)=\frac{1}{x^{2}}, \quad f(x)=\frac{1}{x^{2}}\left[a+b\left(x^{2}+4\right) e^{-x^{2} / 4}\right], \quad m=2 .
$$

The unknown coefficients must be chosen to ensure the good behavior of the solution close to the origin (i.e., $b=-a / 4)$. Similarly,

$$
p(x)=\frac{1}{x^{4}}-\frac{1}{12 x^{2}}, \quad f(x)=\frac{1}{x^{4}}\left[a\left(x^{2}-12\right)+b\left(x^{4}+16 x^{2}+96\right) e^{-x^{2} / 4}\right], \quad m=4,
$$

with $b=a / 8$. Obviously, we can obtain general solutions of this form for all even $m$.

\section{A3. THE RECONNECTION SOLUTION}

The case of most interest physically is $m=1$. This is the only mode which allows finite current at the neutral point, and hence topological reconnection. Although it is not possible to obtain closed form solutions for odd $m$, we can use the series expansion (A5) as an inner approximation to match the outer solution $f_{0}(r)=A / r^{2}$. The result of matching the outer solution to an eight-term inner expansion is shown in Figure $2 a$ (see $\S 4.3)$.

Finally, we mention the scaling of the reconnection rate with resistivity. If we normalize the outer field so that $f_{0}(1)=1$, then the inner solution $f_{I}(r)=a r+O\left(r^{3}\right)$ must achieve the amplitude $1 / r^{2}$ when $r^{2} \simeq \eta$. Thus, $a$ must scale as $\eta^{-3 / 2}$ to maintain a fixed field amplitude on the outer boundary. Accordingly, the current density at the neutral point must build up as $\eta^{-3 / 2}$. This implies that both the ohmic dissipation rate and the flux annihilation rate achieve the superfast scaling $\eta^{-1}$ and $\eta^{-1 / 2}$.

\section{REFERENCES}

Biskamp, D. 1994,Phys. Rep., 4, 237

Craig, I. J. D., Fabling, R. B., Henton, S. M., \& Rickard, G. J. 1995, ApJ, 455, L197

Craig, I. J. D., \& Henton, S. M. 1995, ApJ, 450, 280

Craig, I. J. D., \& McClymont, A. N. 1991, ApJ, 371, L41

. 1993, ApJ, 405, 207

Forbes, T. G., \& Priest, E. R. 1987, Rev. Geophys. Space Phys., 25, 1583
Hassam, A. B. 1992, ApJ,399, 159

Lau, Y.T., \& Finn, J. M.1990, ApJ, 350, 672

Petschek, H. E. 1964, in AAS-NASA Symposium on tiis Physics of Solar Flares, ed. W. N. Hess (NASA SP-50), 425

Priest, E. R., \& Titov, V. S. 1995, Philos. Trans. R. Soc. London, in press Rosenau, P. 1979, Phys. Fluids, 22, 849

Sonnerup, B. U. O., \& Priest, E. R. 1975, J. Plasma Phys., 14, 283 